\title{
Clinical application of a multiplex genetic pathogen detection system remaps the aetiology of diarrhoeal infections in Shanghai
}

Shiwen Wang ${ }^{1,2,3 \dagger}$, Feng Yang ${ }^{1,2,3 \dagger}$, Dong $\mathrm{Li}^{4 \dagger}$, Juanxiu Qin ${ }^{5 \dagger}$, Weiwei Hou ${ }^{4}$, Lian Jiang ${ }^{4}$, Mimi Kong ${ }^{6}$, Yong Wu ${ }^{6}$, Yuchen Zhang ${ }^{6}$, Fuju Zhao ${ }^{1,2,3}$, Yi Fang ${ }^{1,2,3}$, Yingxin Miao ${ }^{1,2,3}$, Lingli Xu' ${ }^{7}$, Jie Chen ${ }^{2,3,8}$, Zhijun Bao ${ }^{2,3,8^{*}}$, Michal A. Olszewski ${ }^{9^{*}}$, Hu Zhao ${ }^{1,2,3^{*}}$ and Yanmei Zhang ${ }^{1,2,3^{*}}$

\begin{abstract}
Background: Culture-based diagnostic methods cannot achieve rapid and precise diagnoses for the identification of multiple diarrhoeal pathogens (DPS). A high-throughput multiplex genetic detection system (HMGS) was adapted and evaluated for the simultaneous identification and differentiation of infectious DPs and a broad analysis of DP infection aetiology.

Results: DP-HMGS was highly sensitive and specific for DP detection compared with culture-based techniques and was similar to singleplex real-time PCR. The uniform level of sensitivity of DP-HMGS for all DPs allowed us to remap the aetiology of acute diarrhoeal infections in Shanghai, correcting incidences of massively underdiagnosed DP species with accuracy approaching that of sequencing-based methods. The most frequent DPs were enteropathogenic Escherichia coli, rotavirus and Campylobacter jejuni. DP-HMGS detected two additional causes of infectious diarrhoea that were previously missed by routine culture-based methods: enterohemorrhagic E. coli and Yersinia enterocolitica. We demonstrated the age dependence of specific DP distributions, especially the distributions of rotavirus, intestinal adenovirus and Clostridium difficile in paediatric patients as well as those of dominant bacterial infections in adults, with a distinct"top 3" pattern for each age group. Finally, the multiplexing capability and high sensitivity of DP-HMGS allowed the detection of infections co-induced by multiple pathogens (approximately 1/3 of the cases), with some DPs preferentially co-occurring as infectious agents.
\end{abstract}

Conclusions: DP-HMGS has been shown to be a rapid, specific, sensitive and appropriate method for the simultaneous screening/detection of polymicrobial DP infections in faecal specimens. Widespread use of DP-HMGS is likely to advance routine diagnostic and clinical studies on the aetiology of acute diarrhoea.

Keywords: Diarrhoeal pathogens (DPs), Rapid screening, High-throughput multiplex genetic detection system (HMGS), Faecal specimens, DP composition, Polymicrobial infection

\footnotetext{
*Correspondence: xinyi8681@sina.com; olszewsm@med.umich.edu; hubertzhao@163.com; 15618653286@163.com

†Shiwen Wang, Feng Yang, Dong Li and Juanxiu Qin contributed to this work equally

1 Department of Laboratory Medicine, Huadong Hospital, Affiliated with Fudan University, Shanghai 200040, China

${ }^{8}$ Department of Gastroenterology, Gerontology Institute of Shanghai,

Affiliated with Huadong Hospital, Affiliated with Fudan University, Shanghai 200040, China

${ }^{9}$ Division of Pulmonary and Critical Care Medicine, Department of Internal Medicine, University of Michigan Health System and Research

Service, VA Ann Arbor Health Systems, Ann Arbor, MI, USA

Full list of author information is available at the end of the article
} 


\section{Background}

Diarrhoeal diseases caused by enteric infections continue to pose a major threat to global health $[1,2]$. With respect to the overall impact on human health, diarrhoea ranks second among all infectious diseases $[3,4]$, with approximately 2 billion incidences of diarrhoeal diseases reported annually in China alone [5]. The World Health Organization (WHO)'s Global Burden of Disease study lists diarrhoeal diseases as one of the leading causes of preventable deaths worldwide [6].

One of the greatest challenges in the diagnosis of diarrhoeal diseases is that a large number of aetiological agents are associated with generally non-specific clinical symptoms [7]. Traditional detection methods for these pathogens in faecal specimens are culture-based methods, immunological detection assays, and molecular diagnostic methods [8]. The culture-based methods, while considered the "gold standard" options for routine diagnosis, are time consuming $[9,10]$. Furthermore, infections caused by microbes with very stringent/specific requirements for culture conditions are likely to be underdiagnosed, especially in cases of polymicrobial infections. The development of molecular diagnostic methods such as real-time PCR, has increased the sensitivity of these methods, but typically, such testing is restricted to a single pathogen per test. Finally, metagenomic approaches using next-generation sequencing, while capable of generating virtually infinite amounts of information, require sophisticated analytical tools and time-consuming analysis and are very costly; thus, these methods cannot be broadly applied in everyday diagnosis of common diarrhoeas. Due to these limitations, precise aetiological diagnosis of enteric infections is not routinely accomplished, resulting in poor therapeutic efficacy and increased risks for the development of drugresistant bacteria and introduction of imbalance in the intestinal microbiota (dysbiosis) [11, 12].

Studies conducted worldwide demonstrate wide variations in the prevalence and composition of causative agents of acute diarrhoea [13-16]. These discrepancies can arise due to non-uniform diagnostic approaches and preferential detection of some pathogens. These broad concerns resulted in the publication of clinical guidelines by the Expert Consensus on Diagnosis and Treatment of Infectious Diarrhoea in Chinese Adults (2013), which recommend aetiological diagnosis to promote rational treatment of diarrhoeal diseases, proper epidemiological studies of these diseases, and prevention of antimicrobial drug resistance $[17,18]$.

Here, we redesign and adapt a high-throughput multiplex genetic detection system (HMGS) to screening faecal specimens for 19 major pathogenic diarrhoeal pathogens (DPs) that cause acute diarrhoeal infections
[19]. A total of 613 faecal specimens were analysed by the DP-HMGS assay, sequencing, and conventional methods (culture-based methods and singleplex real-time PCR) in parallel, and the methods were compared for accuracy and applicability in the generation of epidemiological data.

\section{Methods \\ Ethics statement}

This study was carried out in accordance with the recommendations of the Ethics Committee for Human Studies of Huadong Hospital and registered under Ethics Approval Number 2013-077 with written informed consent from all subjects. All subjects provided written informed consent in accordance with the Declaration of Helsinki.

\section{Diarrhoeal pathogens}

Based on epidemiological investigations, the DPs that most commonly cause diarrhoeal diseases in the Shanghai area were selected as candidates for the DP-HMGS screening assay [20-22]. The six most common viral pathogens that cause outbreaks of gastroenteritis, namely, human astrovirus (HASV), norovirus II (NorV), human adenovirus (HADV), rotavirus A (RoVA), rotavirus $\mathrm{B}(\mathrm{RoVB})$, and rotavirus $\mathrm{C}(\mathrm{RoVC})$, as well as a negative control sapovirus were isolated from clinical specimens at Huadong Hospital, Shanghai, China and verified by sequencing of species-specific, conserved genes. A group of bacterial species that either most commonly cause enteritis and/or induce severe forms of enteritis were obtained from Shanghai Municipal Center for Disease Control \& Prevention (CDC). Standard ATCC strains of the following bacterial species were used: Campylobacter jejuni (C. jejuni) ATCC33560, Shigella ATCC12022, pathogenic Clostridium difficile (C. difficile) ATCC9689, Salmonella enteritidis ( $S$. enteritidis) ATCC31194, Salmonella typhimurium (S. typhimurium) ATCC14028, Vibrio parahaemolyticus (V. parahaemolyticus) ATCC17802, Yersinia enterocolitica (Y. enterocolitica) ATCC23715. From the CDC, we obtained the standard control microbes, namely, Helicobacter pylori (H. pylori) ATCC43504, Pseudomonas aeruginosa ATCC27853, Staphylococcus aureus (S. aureus) ATCC29213, and Escherichia coli (E. coli) ATCC35218. Other common bacterial DP strains selected for DPHMGS testing, including 6 major pathogenic strains of $E$. coli-enterotoxigenic E. coli (ETEC), enterohemorrhagic E. coli (EHEC), enteropathogenic E. coli (EPEC), enteroaggregative $E$. coli (EAEC), enteroinvasive E. coli (EIEC), and E. coli $\mathrm{O} 157$ strain (E. coli $\mathrm{O} 157)$ - as well as the nonpathogenic E. coli strain DH5 (E. coli DH5 $\alpha$ ) and Plesiomonas shigelloides were isolated from clinical specimens 
at Huadong Hospital, Shanghai, China, and verified by sequencing of species-specific conserved genes.

\section{Faecal specimen collection}

Six hundred and thirteen faecal specimens were obtained from outpatients diagnosed with diarrhoea from January 2016 to November 2017, and 30 faecal specimens were obtained from healthy volunteers from Renji Hospital and Children's Hospital, affiliated with Shanghai Jiaotong University; Tongji Hospital, affiliated with Tongji University; and the Centers for Disease Control in Songjiang district in Shanghai. Patients of all ages with symptoms of acute diarrhoea were considered to be eligible for enrolment. As per the ACG clinical guidelines, acute diarrhoea was defined as the occurrence of defecation 3 or more times per $24 \mathrm{~h}$, with abnormal faecal characteristics, such as loose stool, watery stool, mushy stool, mucosal stool and bloody stool, lasting for less than 14 days [23]. The exclusion criteria were diarrhoea caused by medicines, poisons, food allergies food intolerance or other diseases. Patients undergoing antibiotic treatments were also excluded. Fresh whole faecal specimens $(10 \mathrm{~g})$ were collected in sterilized containers containing $2 \mathrm{~mL}$ of normal saline supplemented with recombinant RNase inhibitor (TaKaRa, Japan) to prevent degradation of genetic material from RNA viruses and stored at $-20^{\circ} \mathrm{C}$ within $2 \mathrm{~h}$.

\section{Nucleic acid extraction from faecal specimens}

Total nucleic acid was extracted from a $200 \mu \mathrm{L}$ faecal suspension using the Whole Genome Extraction Kit (Zhongding Biotech Co., Ltd., Ningbo, China) according to the manufacturer's instructions. The extracts were eluted with $100 \mu \mathrm{L}$ of DNase/RNase-free $\mathrm{H}_{2} \mathrm{O}\left(\mathrm{ddH}_{2} \mathrm{O}\right)$. The concentrations of each extract were determined using a Thermo Nanodrop 2000 spectrophotometer (Thermo Fisher Scientific Inc., Waltham, MA, USA). The extracts were stored at $-80^{\circ} \mathrm{C}$ until further analysis.

\section{Cloning and sequencing}

The genomic targets of the selected pathogens were amplified, and the resulting products were purified using the High Pure PCR Product Purification Kit (Roche, Basel, Switzerland) and subsequently ligated into the pMD18-T simple vector. The constructs were transformed into E. coli DH5 $\alpha$, followed by sequencing by Shanghai RuiDi Biological Technology Company. Sequencing was performed using the Sanger method with an ABI 3730XL automated DNA analyser (Applied Biosystems Inc., California, USA). The DNA sequences were verified by a BLAST search of the National Center of Biotechnology Information (NCBI) nucleotide database (http://www.ncbi.nlm.nih.gov/blast) using
DNASTAR Lasergene analysis software (DNASTAR Inc., WI, USA).

\section{Bacterial culture and identification}

Faecal specimen suspensions were mixed briefly and transferred $(1 \mathrm{~mL})$ into a TissueLyser to obtain a uniform suspension (Jingxin Co., Ltd., Shanghai, China) and cultured with Salmonella-Shigella (SS) agar and Columbia blood agar at $37^{\circ} \mathrm{C}$ for $24 \mathrm{~h}$ for Shigella and S. typhimurium. C. jejuni were cultured with charcoal cefoperazone deoxycholate agar (CCDA) under microaerophilic conditions at $37^{\circ} \mathrm{C}$ for $24 \mathrm{~h}$. The specimens were also inoculated into selective enrichment broth at $37{ }^{\circ} \mathrm{C}$ for $24 \mathrm{~h}$, followed by subculturing on thiosulphate-citrate-bile salts-sucrose (TCBS) agar for culturing Vibrio species. Colonies of Vibrio parahaemolyticus and Vibrio minicus (green colonies on TCBS) and Vibrio cholerae and Vibrio fluvialis (yellow colonies on TCBS) were identified by matrix-assisted laser desorption/ionization time-of-flight mass spectrometry (BioMerieux, Lyon, France). Different serotypes of $E$. coli were inoculated onto Columbia blood agar and cultured at $37^{\circ} \mathrm{C}$ for $24 \mathrm{~h}$. Y. enterocolitica was inoculated into MacConkey agar and cultured at $28{ }^{\circ} \mathrm{C}$ for 48 h. C. difficile was inoculated into cycloserine cefoxitin fructose agar (CCFA) under anaerobic conditions a $37^{\circ} \mathrm{C}$ for $24 \mathrm{~h}$.

\section{Viral identification by singleplex real-time PCR}

The Real-Time PCR Kit (BioPerfectus Technologies, Taizhou, China) was used to detect adenovirus, which is a DNA virus. Three reverse transcription PCR kits (BioPerfectus Technologies, Taizhou, China; including reverse transcriptase) were used to detect the RNA viruses norovirus, rotavirus and astrovirus. Four singleplex PCRs were conducted in a real-time PCR system (7500 realtime PCR system; ABI, California, USA) with software version 2.3. All procedures were conducted following the manufacturer's instructions.

\section{Primer design}

The 22 pairs of primers targeting the species-specific conserved genomic fragments of the selected DPs were designed to include 13 bacterial DPs (listed above), 6 viral DP (listed above), a human internal RNA control gene (hum_RNA) beta-2 microglobulin $(B 2 M)$, a human internal DNA control gene (hum_DNA) ribonuclease P (RNaseP), and a systematic internal control (IC). Hundreds of sequences were downloaded from NCBI and analysed using Vector NTI to identify the most highly conserved gene targets specific for each individual DP type. The primers for amplification of the highly conserved regions were designed using DNASTAR software (DNASTAR Inc., Madison, WI, USA) and Primer 
Premier 5.0 software (Premier Biosoft International, Palo Alto, CA, USA). All the primers were synthesized and purified by Invitrogen ${ }^{\mathrm{TM}}$, China. These gene-specific primers were designed and optimized by applying the following criteria: homogeneity of primer sequences; amplification product sizes ranging from 100 to $350 \mathrm{bp}$, with at least 3-base-pair size differences between each fragment; absence of significant dimer formation between different primers; and absence of non-specific products with each pair of gene-specific primers.

The specific primer sets used in the DP-HMGS molecular detection assay and the corresponding amplicon sizes are all listed in Additional file 1: Table S1. The specificity of each single pair of primers was verified by singleplex PCR using templates containing all the corresponding extracted nucleic acids from each DP, and confirmed by Sanger sequencing. All the primers used for Sanger sequencing are listed in Additional file 2: Table S2. The primer pairs that generated amplification products with a single specific DP-HMGS peak but no non-specific peaks were selected (shown in Additional file 3: Figure S1A-Q).

\section{Setup of the DP-HMGS assay}

Each DP-HMGS reaction contained $2 \mu \mathrm{L}$ of $5 \times$ PCR buffer, $0.35 \mu \mathrm{L}$ of $10 \mu \mathrm{M}$ dNTPs, $0.25 \mu \mathrm{L}$ of $25 \mathrm{mM}$ $\mathrm{MgCl}_{2}, 0.4 \mu \mathrm{L}$ of $5 \mathrm{U} / \mu \mathrm{L}$ enzyme mix (Taq polymerase and reverse transcriptase), $0.1 \mu \mathrm{L}$ of $1 \mathrm{U} / \mu \mathrm{L}$ anti-contamination enzyme UDG (uracil DNA glycosylase; TaKaRa, Japan) [24], $1 \mu \mathrm{M}$ each of the forward and reverse primers, and $2.5 \mu \mathrm{L}$ of plasmid; the amount of plasmid template for each target pathogen in the HMGS assay ranged from 5 to $50 \mathrm{ng}$. $\mathrm{ddH}_{2} \mathrm{O}$ was added to the PCR to attain a final volume of $10 \mu \mathrm{L}$. The PCR mixture was incubated as follows: $25{ }^{\circ} \mathrm{C}$ for $5 \mathrm{~min} ; 50{ }^{\circ} \mathrm{C}$ for $30 \mathrm{~min} ; 95{ }^{\circ} \mathrm{C}$ for $15 \mathrm{~min} ; 35$ cycles of $94{ }^{\circ} \mathrm{C}$ for $30 \mathrm{~s}, 60^{\circ} \mathrm{C}$ for $30 \mathrm{~s}$, and $72{ }^{\circ} \mathrm{C}$ for $1 \mathrm{~min} ; 72{ }^{\circ} \mathrm{C}$ for $15 \mathrm{~min}$.

\section{Separation by capillary electrophoresis and fragment analysis}

Following the amplification step, $1 \mu \mathrm{L}$ of the reaction product was added to $9 \mu \mathrm{L}$ of highly deionized (Hi-Di) formamide along with $0.25 \mu \mathrm{L}$ of DNA Size Standard 500 (AB Sciex, USA). The Applied Biosystems 3500DX genetic analysis system (Applied Biosystems, California, USA) was then used to analyse the PCR products based on size separation using high-resolution capillary gel electrophoresis. The peak height for each PCR product was reported in the electropherogram, and the reaction was considered to be positive when the dye signal was greater than 300 relative fluorescence units ( $\mathrm{rfu}$ ). $\mathrm{ddH}_{2} \mathrm{O}$ was used as a negative control throughout the experimental process.
Establishment and optimization of the DP-HMGS assay Multiple sets of primers and reaction parameters were used to optimize the performance of the DP-HMGS assay in a single reaction. The main optimization principle was to keep all amplicons that had similar amplification efficiency ranges and exhibited the gene-specific target amplicons. Primer sequences, concentrations and ratios were optimized so that each DP signature could be amplified specifically without cross-interaction. Additionally, the annealing temperature was optimized using the temperature gradient descent method (using chimeric primers, with temperatures from 50 to $65{ }^{\circ} \mathrm{C}$ ). Other reaction parameters, such as buffer, enzyme, and reaction time, were also systematically optimized. The primers for the human internal RNA control gene $B 2 M$ and human internal DNA control gene RNaseP were included in the DP-HMGS PCR primer mix. Detections of these two genes in the samples indicated that no significant nucleic acid degradation had occurred during specimen handling/storage. Additionally, a modified fragment of the kanamycin resistance gene (Kanr) was inserted into the pcDNA3.1 vector to generate a fusion plasmid that served as internal control for the detection system. The fusion plasmid $\left(1.5 \times 10^{5}\right.$ copies in $\left.3 \mu \mathrm{L}\right)$ was added to the $200 \mu \mathrm{L}$ faecal suspension, immediately prior to nucleic acid extraction, to monitor the extraction and the DP-HMGS reaction. The appearance of all 3 internal control peaks in the DP-HMGS trace confirmed that the sample RNA and DNA had good integrity and underwent efficient extraction, processing, and amplification.

\section{Sensitivity, specificity and accuracy of the DP-HMGS assay}

The sensitivity of the DP-HMGS assay for each pathogen was tested by serial tenfold dilutions of plasmids. Serial tenfold dilutions of 22 plasmids using equal amounts of templates were used to test the simultaneous detection limit of the DP-HMGS for all pathogens. The specificity of the DP-HMGS assay in detecting pathogens in a microbiologically diverse gastrointestinal environment was tested using plasmids from 19 positive DPs from our panel combined with DNA from 7 negative control pathogen species expected to be present in the GI tract, namely, H. pylori, E. coli DH5 $\alpha$, Pseudomona aeruginosa, S. aureus, Plesiomonas shigelloides, E. coli and sapovirus (Additional file 4: Figure S2). To assess the accuracy, different amounts of three pathogen-associated plasmids ( $S$. enteritidis, $1 \times 10^{3}$ copies; HADV, $1 \times 10^{5}$ copies; EHEC, $1 \times 10^{4}$ copies) were randomly selected from the 19 types of DPs and mixed for testing with the DP-HMGS assay, and the results were compared with those of the single-template HMGS assay. The plasmids containing genes of the 7 negative control species were then mixed with plasmids containing genes from selected pathogenic species (S. enteritidis, HADV 
and EHEC) to further test the ability of the DP-HMGS assay to identify polymicrobial infections in microbiologically diverse environments. The reaction system setup and detection were performed as described above.

\section{Data analysis and statistics}

The sensitivity and specificity of the diagnostic tests were calculated according to the following formulas: $\mathrm{SE}=\mathrm{TP} /(\mathrm{TP}+\mathrm{FN}) \times 100 ; \quad \mathrm{SP}=\mathrm{TN} /(\mathrm{TN}+\mathrm{FP}) \times 100$; the positive predictive value (PPV) and negative predictive value $(\mathrm{NPV})$ were calculated as follows: $\mathrm{PPV}=\mathrm{TP} / \mathrm{P}$; $\mathrm{NPV}=\mathrm{TN} / \mathrm{N}$ (FN: false negative; FP: false positive; $\mathrm{N}$ : negative; P: positive; SE: Sensitivity; SP: specificity; TN: true negative; TP: true positive). Among these variables, TP refers to the number of samples that were positively detected by conventional methods (culture-based methods and singleplex real-time PCR) or DP-HMGS and the Sanger sequencing method. TN refers to the number of samples that gave negative results with conventional methods (culture-based methods and singleplex real-time PCR) or DP-HMGS and the Sanger sequencing method. FP refers to the number of samples that were positively detected by conventional methods (culture-based methods and singleplex real-time PCR) or DP-HMGS but gave negative results with the Sanger sequencing method. FN refers to the number of samples that gave negative results with conventional methods (culture-based methods and singleplex real-time PCR) or DP-HMGS but were positively detected by the Sanger sequencing method. The data were statistically analysed by the $\chi^{2}$ test using the Stata statistical software package, version 12.0 (Stata Corp College Station, TX, USA). The DP distribution of different groups was analysed by the Mann-Whitney rank-sum test for two variables and the Kruskal-Wallis $\mathrm{H}$ test for more than two variables. All of the above hypothesis tests were two-sided, and a twotailed p-value of 0.05 or less was considered to indicate statistical significance.

\section{Results}

The optimized DP-HMGS assay allows for concurrent amplification of the DNA signature and simultaneous detection of up to $19 \mathrm{DPs}$ in a single multiplex reaction

The 19 pairs of microbe-specific primers were designed for detection of the genetic signatures of these primers, and 3 primer sets were designed for quality control, as described in the Methods section (Additional file 1: Table S1). Following the testing of individual primer pairs for the detection of specific DP DNA signatures (Additional file 3: Figure S1), we evaluated the performance of all the primers combined in a single multiplex PCR (Fig. 1a). While most of the DP target signals were detected, substantial differences among the amplification signal intensities were initially observed; in addition, peaks for Y. enterocolitica and E. coli O157, the DNA templates for which were included in the 22-plex assay, were absent. To offset these differences in the amplification efficiencies of individual templates, the primer sequences and reaction parameters were further adjusted. This optimization process enabled us to detect all the pathogenic signatures (Fig. 1b) and to generate peaks for all the DNA targets with intermediate signal levels, which allowed for a potential increase or decrease in the signal based on variations among individual samples. Thus, a single multiplex-PCR-based DP screening assay (DP-HMGS) was developed and optimized for simultaneous detection of 19 major DPs with relatively uniform sensitivity.

\section{The DP-HMGS assay is highly specific and sensitive for the detection of DP signatures}

To exclude the possibility of FP detection, nineteen pathogen templates and 3 controls were used as positive controls to test the specificity of the DP-HMGS assay, which produced specific amplification signals for the signature DNA from each target pathogen (Additional file 4: Figure S2A). This result was confirmed using DNA from 7 microbial species for which specific primers were not included in our DP-HMGS screening (H. pylori, $S$. aureus, Pseudomonas aeruginosa, Plesiomonas shigelloides, E. coli $\mathrm{DH} 5 \alpha$, E. coli, and sapovirus); the controls did not exhibit any specific amplification peaks, similar to the negative control $\mathrm{ddH}_{2} \mathrm{O}$ (Additional file 4: Figure S2B-I).

Next, to determine the limit of detection in terms of copy number of the DP signature gene, we tested the sensitivity of DP-HMGS using various concentration ranges of the specific pathogen templates. Twenty-two plasmid pairs to amplify the DP target and control genes were generated and tested using a broad range of known concentrations. The assay was extremely reliable in the detection of $10^{3}-10^{5}$ copies of all the microbial genes, demonstrating that the sensitivity for simultaneous detection of all pathogens by the DP-HMGS assay was at least $1 \times 10^{3}$ copies $/ \mu \mathrm{L}$ (Fig. 2), while further titration to $1 \times 10^{2}$ gene copies/ $\mu \mathrm{L}$ resulted in the generation of relatively small peaks, which for some pathogens were below the cut-off fluorescence signal of $300 \mathrm{rfu}$. Thus, reliable detection of specific DP signatures by DP-HMGS can consistently occur at a concentration of $1 \times 10^{3}$ copies/ $\mu \mathrm{L}$.

\section{DP-HMGS can accurately detect individual DPs in polymicrobial mixtures}

DP-HMGS was designed specifically for application in the detection of polymicrobial infections. To demonstrate this capability, a mixture of $3 \mathrm{DP}$-associated plasmids $-S$. 


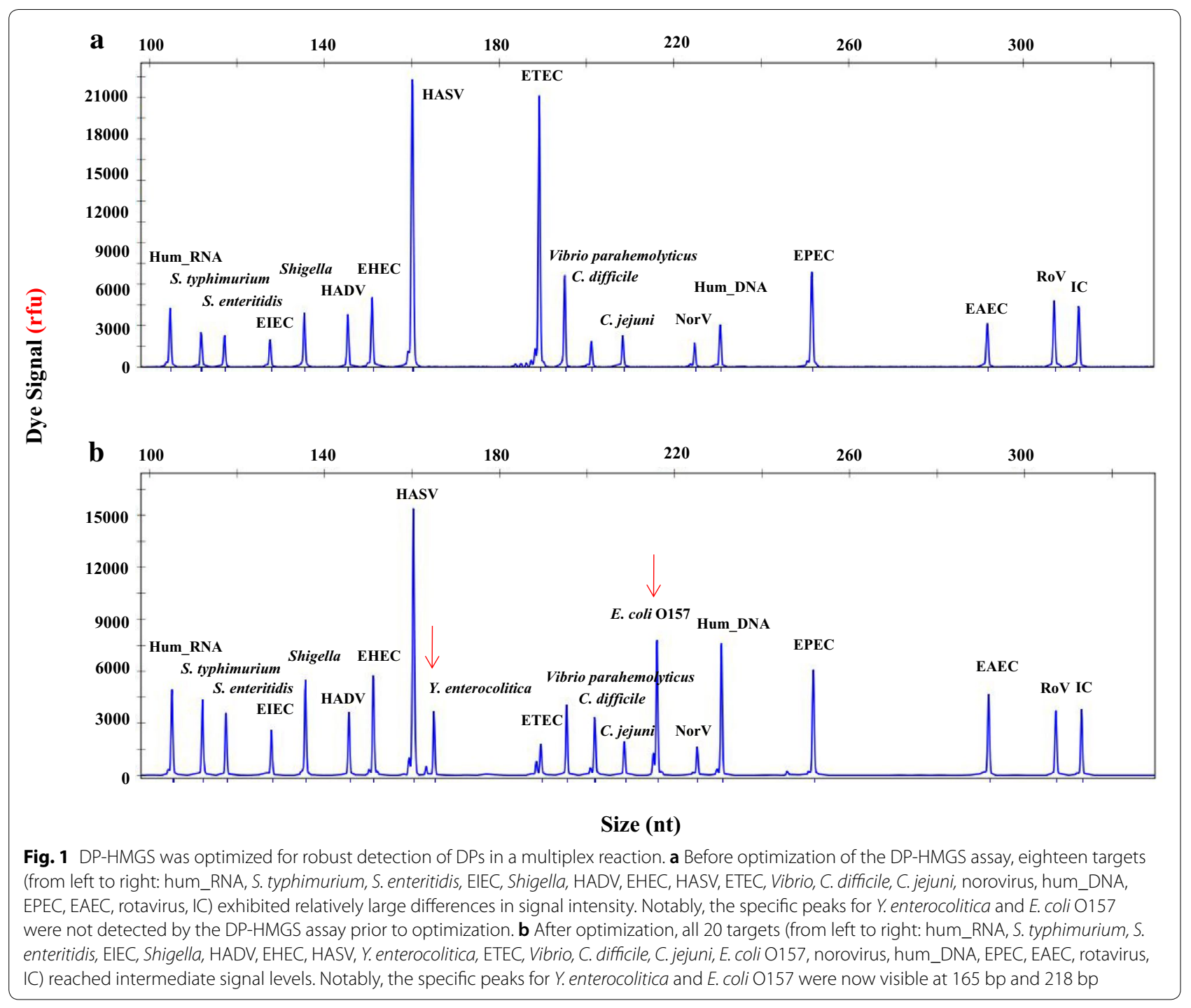

enteritidis, $1 \times 10^{3}$ copies $/ \mu \mathrm{L}$; HADV, $1 \times 10^{5}$ copies/ $\mu \mathrm{L}$; and EHEC, $1 \times 10^{4}$ copies $/ \mu \mathrm{L}$-were combined and screened by the DP-HMGS assay. Three specific amplification peaks (S. enteritidis, 117.58 bp; HADV, 145.61 bp; EHEC, 151.19 bp) were observed, as shown in Fig. 3a. Again, the addition of the 7 negative control microbial species did not interfere with the generation of the specific S. enteritidis, HADV and EHEC peaks, and no FP signals were induced in the presence of these negative control microbes (Fig. 3b). One possible pitfall of multiplex assays is interference by a strong signal with the peaks of a weak signal, which could be expected in the case of polymicrobial infections with a highly abundant DP accompanied by less abundant DPs. We assessed whether microbial DNA with high template quantities could interfere with the detection of low-abundance DNA signatures. The results indicated that the signal magnitude generated in the DP-HMGS assay was identical whether all 3 DNA templates were tested in combination or individually (Fig. 3c-e). Thus, DP-HMGS robustly detected multiple DP signatures without interference by the signal for the dominant DNA template with those of low-abundance DNA templates.

\section{DP-HMGS can directly detect specific microbial signatures in faecal specimens}

Before large-scale validation studies were conducted, we sought to determine whether DP-HMGS would perform "as expected" with healthy and diarrhoeal faecal specimens. A faecal specimen with confirmed $C$. difficile infection was compared against faecal specimens from healthy volunteers. The samples were simultaneously subjected to full genetic material extraction and a full cycle of DP-HMGS screening. The infected 


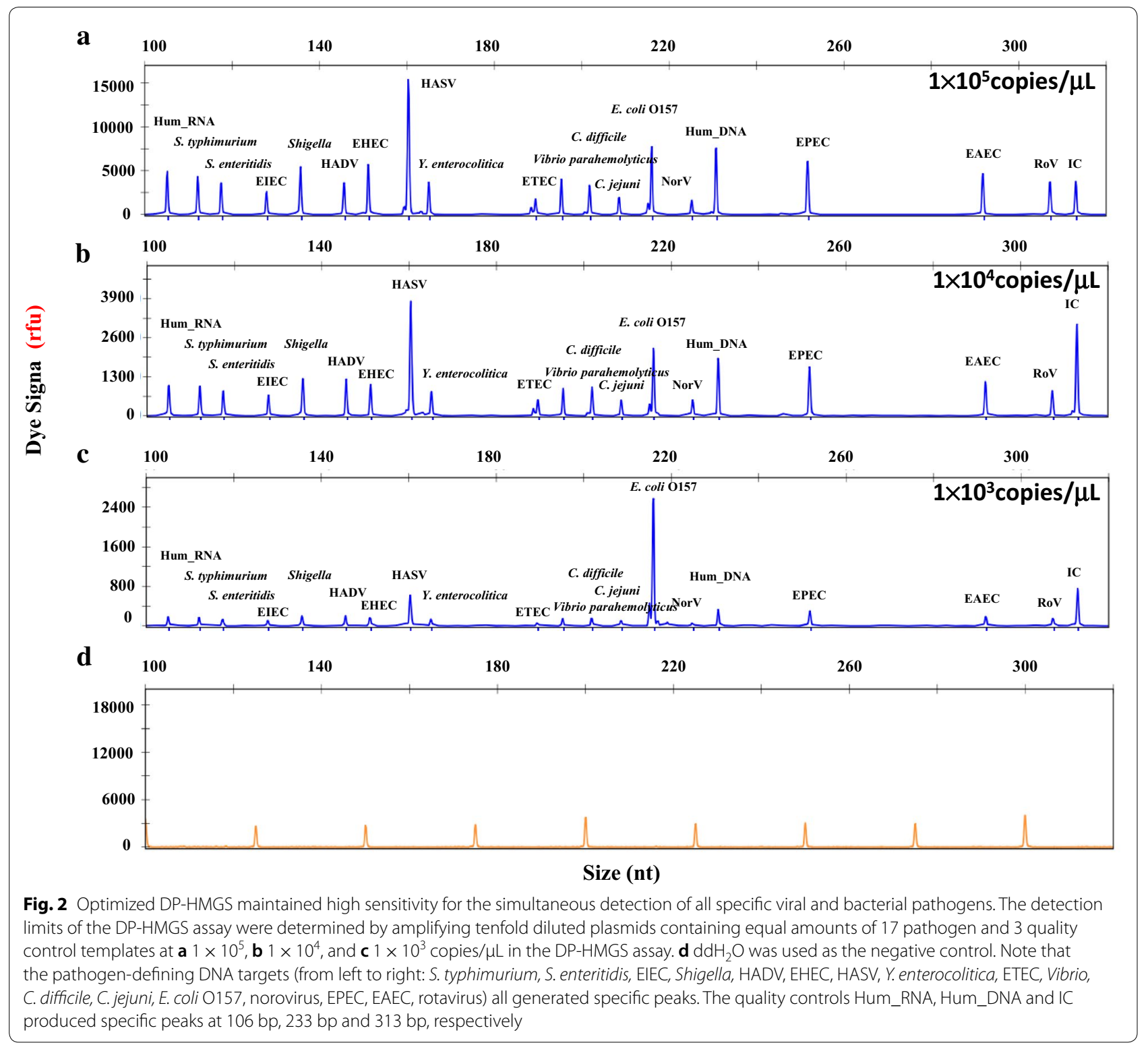

faecal specimens processed by DP-HMGS successfully exhibited the specific amplification signals: the target DP signal (C. difficile) and signals for all three quality control genes, namely, B2 M, RNaseP, and IC (Additional file 5: Figure S3A). The non-diarrhoeal faecal specimens did not produce any signals except for those corresponding to the 3 quality control products (Additional file 5: Figure S3B). The concurrently run negative control reaction $\left(\mathrm{ddH}_{2} \mathrm{O}\right)$ did not produce any peaks, except for the expected peak for IC (Additional file 5: Figure $\mathrm{S} 3 \mathrm{C}$ ). These tests were repeated with consistent results using faecal specimens with other confirmed DP infections (data not shown). Thus, the results of DP-HMGS analysis of faecal specimens were similar to those obtained under the optimized conditions using microbial DNA and plasmids, motivating us to perform further studies to validate DP-HMGS performance in clinical settings.

\section{The clinical study demonstrated the uniformly high} sensitivity and specificity of DP-HMGS in the detection of DPs in faecal specimens, in contrast to the non-uniform sensitivity of conventional methods

To test the performance of DP-HMGS in clinical applications, we conducted a clinical study by analysing faecal specimens of 30 healthy volunteers and 613 hospital patients with suspected infectious diarrhoea. Among these patients, 319 (52.0\%) were males and 294 (48.0\%) 


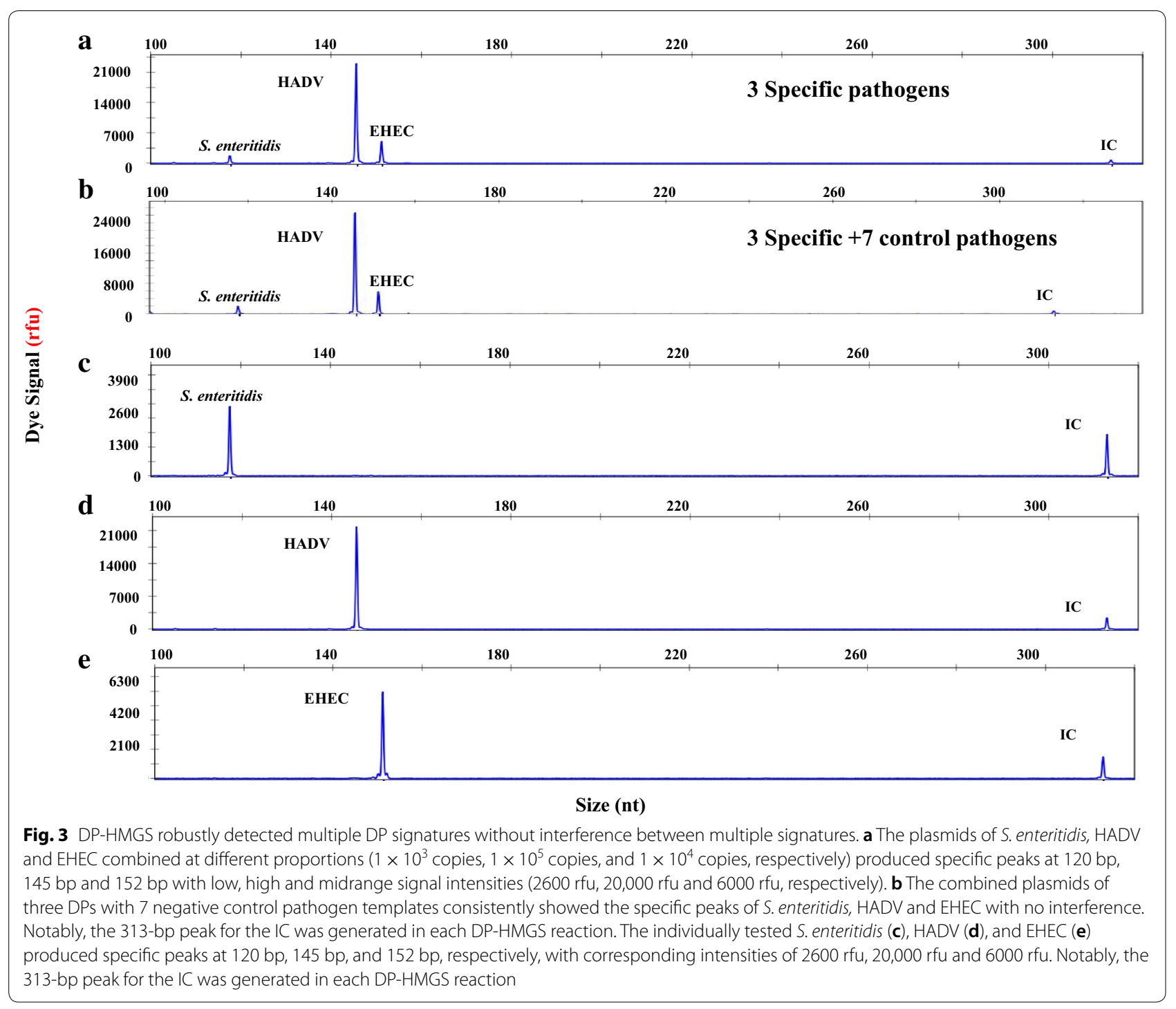

were females. Loose stool was the most common symptomatic characteristic (37.4\%), followed by watery stool (34.3\%), mushy stool (22.0\%), mucosal stool (5.4\%) and bloody stool $(0.9 \%)$. All of the faecal specimens collected from the recruited patients were assayed and processed as outlined in Fig. 4. For all the faecal specimens, the culture-based method was used to identify possible bacterial pathogens. Nucleic acids were extracted from the faecal specimens to perform singleplex real-time PCR for virus detection and DP-HMGS analysis. For bacterial identification, DP-HMGS was compared with the culture-based method. For virus identification, DP-HMGS was compared with singleplex real-time PCR. The specimens with inconsistent results between DP-HMGS and the culture-based method were further verified by conventional PCR and Sanger sequencing, which is considered the "gold standard" for gene detection $[25,26]$.

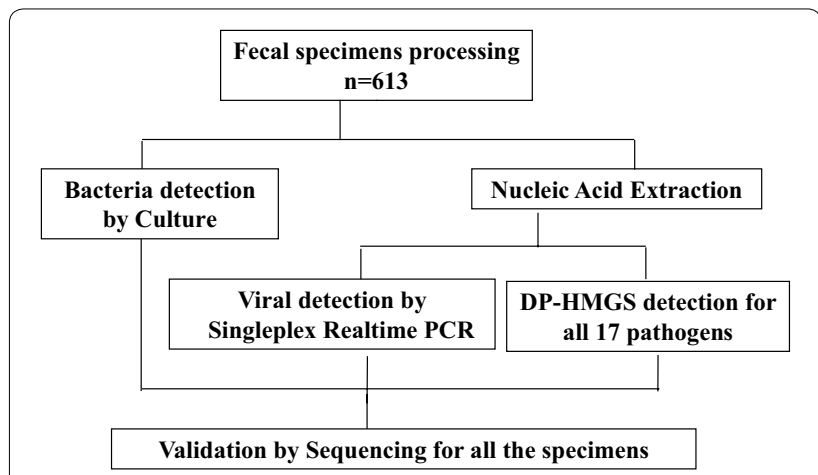

Fig. 4 Workflow for faecal specimen processing. A schematic workflow chart depicting the processing of 613 faecal specimens for analysis, comparing conventional methods and DP-HMGS

The primer sequences for conventional PCR and product sizes for Sanger sequencing are shown in Additional file 2: Table S2. Finally, the extracted nucleic acids were 
used for DNA sequencing as the most stringent reference to calculate sensitivities, specificities, and the predictive values of DP-HMGS, the conventional culture-based methods (for bacteria), and singleplex real-time PCR (for viruses).

DP-HMGS testing of faecal specimens from healthy individuals produced only the specific internal control peaks of Hum_RNA, Hum_DNA and IC, without any DP-specific peaks (data not shown). There were 345 positive DP specimens among the 613 faecal specimens validated by DNA sequencing, and 494 DPs were detected from these 345 positive specimens. The results consistently showed that the sensitivity and NPV of DP-HMGS were higher than those of the culture-based method for all bacteria (Tables 1 and 2). For virus detection, the sensitivity of DP-HMGS was as high as that of singleplex real-time PCR. The specificity and PPV of DP-HMGS were lower than those of the culture-based method for bacteria but were similar to those of singleplex realtime PCR for viruses (Tables 1 and 2). Thus, the efficacies of culture-based methods for bacterial pathogens were highly variable compared to the relatively uniform and sensitive performance of DP-HMGS. The single PCR assays conducted for the detection of a single viral pathogen were comparable with DP-HMGS.

\section{DP-HMGS corrects inaccurate conclusions regarding major causes of infectious diarrhoeas by summarizing the combined outcomes of conventional detection methods of major DPs}

High disparity among the detection parameters of various DPs using conventional detection methods (Table 1) prompted a careful analysis of the detection frequencies of the individual microbial species. Comparison of the detection rates by the culture-based method and DPHMGS for 13 bacterial pathogens demonstrated that the sensitivity of DP-HMGS for all diarrhoeal bacteria was substantially higher than that of the culture-based method (Fig. 5a). The sensitivity of culture-based detection of different bacterial species was rather low (from 0 to $57.5 \%)$. Specifically, E. coli O157 (16.7\%), C. difficile (21.4\%), and EIEC (25\%) exhibited positive detection rates, while EHEC and Y. enterocolitica were not detected at all. This sensitivity was in contrast to the comparable sensitivity of the detection of viral infection, for which individual PCR is a standard detection method. Because bacterial culture-based detection of bacterial DPs and PCR-based detection of viral DPs continue to be widely used to assess DP incidence in clinical studies [20, 27], we suspected that the marked disparity in the detection rates of these methods would substantially alter the conclusions regarding the epidemiological impact of each pathogen. We found that the significant disparity in the detection rates of viral versus bacterial pathogens and the highly variable sensitivities of culture-based detection for individual bacterial species distorted the conclusion regarding the relative importance of each pathogenic group (Fig. 5b) and individual DP species (Fig. 5c). Furthermore, the use of DP-HMGS resulted in complete remapping of the aetiological profile of the studied population with infectious diarrhoeas (Fig. 5c), with frequencies very similar to those obtained by sequencing (not shown). In addition to detecting marked changes in the distribution of DPs, DP-HMGS detected EHEC and $Y$. enterocolitica, which were completely missed by the culture-based detection approach. Collectively, these data show that the uniformly high sensitivity of detection achieved by DP-HMGS allowed the correction of epidemiological conclusions regarding major causes of infectious diarrhoeas, highlighting the prospect of using DP-HMGS as a tool for future clinical studies.

\section{The DP frequency distribution exhibited significant age variation}

We expanded the analysis of the epidemiological distribution of DP frequencies in our patient cohort, analysing the incidence of DP infections in age- and gender-grouped subpopulations by DP-HMGS (Table 3). The epidemiological analysis demonstrated that the most common infectious bacterial pathogens were EPEC (24.6\%), C. jejuni (7.2\%), S. typhimurium (6.9\%) and Vibrio (6.5\%). Rotavirus was the most common virus and was identified in $9.3 \%$ of the cases of DP infection, while other viruses, including norovirus (6.2\%), adenovirus (2.0\%), and astrovirus (0.7\%), were responsible for a smaller portion of the infections. We observed significant differences in the distribution of DPs within age groups, and the results revealed that the occurrence of most of the infections was age dependent. The most dramatic, age-related differences were observed between total bacterial and viral infections; a major change in the infection profiles was observed between children and adults (Table 3, Fig. 6a). Viral pathogens were responsible for the largest number of detected cases in the youngest ( $\leq 19$ years old) age group (53.9\% cases, 63 out of 117$)$, while bacteria accounted for only $35 \%$ of the cases (41 out of 117). This trend in the age distribution of viral infection was driven entirely by the dynamics of rotavirus and adenovirus infections (Fig. 6b), which together accounted for $87.3 \%$ ( 55 out of 63 ) of the viral infections in young patients and nearly $47.0 \%$ (55 out of 117) of all the specimens in this age group but were only sporadically detected or absent in the remaining age groups (Table 3 ). In addition to high incidences of rotavirus and adenovirus, we observed a significant peak for a single bacterial DP, namely, the pathogenic C. difficile, (Fig. 6b) in these paediatric patients. Further analysis revealed that most of the rotavirus $(75.0 \%, 36 / 48)$, 
Table 1 Comparison of conventional methods and Sanger sequencing for the detection of individual DPs

\begin{tabular}{|c|c|c|c|c|c|c|c|c|}
\hline \multirow[t]{2}{*}{ Bacteria } & \multirow[t]{2}{*}{ Culture } & \multicolumn{2}{|c|}{ Sequencing } & \multirow[t]{2}{*}{ Sensitivity } & \multirow[t]{2}{*}{ Specificity } & \multirow[t]{2}{*}{ PPV } & \multirow[t]{2}{*}{ NPV } & \multirow[t]{2}{*}{ Accuracy } \\
\hline & & + & - & & & & & \\
\hline \multirow[t]{2}{*}{ Vibrio } & + & 23 & 0 & 0.575 & 1.000 & 1.000 & 0.971 & 0.972 \\
\hline & - & 17 & 573 & & & & & \\
\hline \multirow[t]{2}{*}{ S. typhimurium } & + & 17 & 0 & 0.415 & 1.000 & 1.000 & 0.960 & 0.961 \\
\hline & - & 24 & 572 & & & & & \\
\hline \multirow[t]{2}{*}{ S. enteritis } & + & 2 & 0 & 0.333 & 1.000 & 1.000 & 0.993 & 0.993 \\
\hline & - & 4 & 607 & & & & & \\
\hline \multirow[t]{2}{*}{ Shigella } & + & 3 & 0 & 0.333 & 1.000 & 1.000 & 0.990 & 0.990 \\
\hline & - & 6 & 604 & & & & & \\
\hline \multirow[t]{2}{*}{ C. difficile } & + & 6 & 0 & 0.214 & 1.000 & 1.000 & 0.964 & 0.964 \\
\hline & - & 22 & 585 & & & & & \\
\hline \multirow[t]{2}{*}{ C. jejuni } & + & 12 & 0 & 0.267 & 1.000 & 1.000 & 0.946 & 0.947 \\
\hline & - & 33 & 578 & & & & & \\
\hline \multirow[t]{2}{*}{ Y. enterocolitica } & + & 0 & 0 & 0.000 & 1.000 & - & 0.998 & 0.998 \\
\hline & - & 1 & 612 & & & & & \\
\hline \multirow[t]{2}{*}{ EPEC } & + & 49 & 0 & 0.325 & 1.000 & 1.000 & 0.819 & 0.834 \\
\hline & - & 102 & 462 & & & & & \\
\hline \multirow[t]{2}{*}{ ETEC } & + & 11 & 0 & 0.379 & 1.000 & 1.000 & 0.970 & 0.971 \\
\hline & - & 18 & 584 & & & & & \\
\hline \multirow[t]{2}{*}{ EAEC } & + & 8 & 0 & 0.296 & 1.000 & 1.000 & 0.969 & 0.969 \\
\hline & - & 19 & 586 & & & & & \\
\hline \multirow[t]{2}{*}{ EIEC } & + & 2 & 0 & 0.250 & 1.000 & 1.000 & 0.990 & 0.990 \\
\hline & - & 6 & 605 & & & & & \\
\hline \multirow[t]{2}{*}{ EHEC } & + & 0 & 0 & 0.000 & 1.000 & - & 0.993 & 0.993 \\
\hline & - & 4 & 609 & & & & & \\
\hline \multirow[t]{2}{*}{ E. coli O157 } & + & 1 & 0 & 0.167 & 1.000 & 1.000 & 0.992 & 0.992 \\
\hline & - & 5 & 607 & & & & & \\
\hline Viruses & \multicolumn{8}{|c|}{ Singleplex real-time PCR } \\
\hline \multirow[t]{2}{*}{ Norovirus } & + & 31 & 2 & 1.000 & 0.997 & 0.939 & 1.000 & 0.997 \\
\hline & - & 0 & 580 & & & & & \\
\hline \multirow[t]{2}{*}{ Rotavirus } & + & 55 & 3 & 1.000 & 0.995 & 0.948 & 1.000 & 0.995 \\
\hline & - & 0 & 555 & & & & & \\
\hline \multirow[t]{2}{*}{ Adenovirus } & + & 8 & 1 & 1.000 & 0.998 & 0.888 & 1.000 & 0.995 \\
\hline & - & 0 & 604 & & & & & \\
\hline \multirow[t]{2}{*}{ Astrovirus } & + & 4 & 0 & 1.000 & 1.000 & 1.000 & 1.000 & 0.997 \\
\hline & - & 0 & 609 & & & & & \\
\hline
\end{tabular}

Outcomes of conventional detection methods: the results of culture-based methods for bacteria and singleplex real-time PCR for viruses were compared to those of Sanger sequencing. Sensitivity $=T P /(T P+F N) \times 100$, Specificity $=T N /(T N+F P) \times 100 ;$ PPV and NPV were calculated as follows: PPV $=T P / P, N P V=T N / N$. Notably, the sensitivity of the culture-based method was very low and highly variable for various species, while the outcomes of PCR-based detection of viruses were consistent with the sequencing method

$D P-H M G S$ diarrheal pathogens high-throughput multiple genetic detection system, PPV positive predict value, NPV negative predict value

adenovirus $(41.7 \%, 5 / 12)$ and pathogenic $C$. difficile $(66.7 \%$, $8 / 12$ ) were detected in the $0-3$-year-old age group. In contrast, bacterial infections predominated in all the adult age groups (20 years old and above), accounting for 63.6$79.8 \%$ of the cases, while viral infections accounted for approximately $5.8-12.7 \%$ of the cases at these age intervals (Fig. 6b, c). Predominant in all the adult age groups were 4 bacterial DPs, namely, Vibrio, S. typhimurium, EPEC, and ETEC (Fig. 6c, d). With the exception of EPEC, which was the most common in all the adult groups, the frequencies of the DPs varied in all the age groups, as exemplified by the "top 3" DPs for each age interval (Fig. 6d). Finally, the incidences of individual DP infections between the gender groups were similar for most pathogens; however, Shigella 
Table 2 DP-HMGS exhibited uniformly high sensitivity of DP detection compared to the sequencing method

\begin{tabular}{|c|c|c|c|c|c|c|c|c|}
\hline \multirow[t]{2}{*}{ Pathogens } & \multirow[t]{2}{*}{ HMGS } & \multicolumn{2}{|c|}{ Sequencing } & \multirow[t]{2}{*}{ Sensitivity } & \multirow[t]{2}{*}{ Specificity } & \multirow[t]{2}{*}{ PPV } & \multirow[t]{2}{*}{ NPV } & \multirow[t]{2}{*}{ Accuracy } \\
\hline & & + & - & & & & & \\
\hline \multirow[t]{2}{*}{ Vibrio } & + & 39 & 1 & 0.975 & 0.998 & 0.975 & 0.998 & 0.997 \\
\hline & - & 1 & 572 & & & & & \\
\hline \multirow[t]{2}{*}{ S. typhimurium } & + & 41 & 1 & 1.000 & 0.998 & 0.976 & 1.000 & 0.998 \\
\hline & - & 0 & 571 & & & & & \\
\hline \multirow[t]{2}{*}{ S. enteritis } & + & 4 & 2 & 0.667 & 0.997 & 0.667 & 0.997 & 0.993 \\
\hline & - & 2 & 605 & & & & & \\
\hline \multirow[t]{2}{*}{ Shigella } & + & 9 & 1 & 1.000 & 0.998 & 0.900 & 1.000 & 0.998 \\
\hline & - & 0 & 603 & & & & & \\
\hline \multirow[t]{2}{*}{ C. difficile } & + & 28 & 0 & 1.000 & 1.000 & 1.000 & 1.000 & 1.000 \\
\hline & - & 0 & 585 & & & & & \\
\hline \multirow[t]{2}{*}{ C. jejuni } & + & 44 & 0 & 0.978 & 1.000 & 1.000 & 0.998 & 0.998 \\
\hline & - & 1 & 568 & & & & & \\
\hline \multirow[t]{2}{*}{ Y. enterocolitica } & + & 1 & 0 & 1.000 & 1.000 & 1.000 & 1.000 & 1.000 \\
\hline & - & 0 & 612 & & & & & \\
\hline \multirow[t]{2}{*}{ EPEC } & + & 151 & 0 & 1.000 & 1.000 & 1.000 & 1.000 & 1.000 \\
\hline & - & 0 & 462 & & & & & \\
\hline \multirow[t]{2}{*}{ ETEC } & + & 29 & 2 & 1.000 & 0.997 & 0.935 & 1.000 & 0.997 \\
\hline & - & 0 & 582 & & & & & \\
\hline \multirow[t]{2}{*}{ EAEC } & + & 27 & 0 & 1.000 & 1.000 & 1.000 & 1.000 & 1.000 \\
\hline & - & 0 & 586 & & & & & \\
\hline \multirow[t]{2}{*}{ EIEC } & + & 8 & 1 & 1.000 & 0.998 & 0.889 & 1.000 & 0.998 \\
\hline & - & 0 & 604 & & & & & \\
\hline \multirow[t]{2}{*}{ EHEC } & + & 4 & 0 & 1.000 & 1.000 & 1.000 & 1.000 & 1.000 \\
\hline & - & 0 & 609 & & & & & \\
\hline \multirow[t]{2}{*}{ E. coli O157 } & + & 6 & 2 & 1.000 & 0.997 & 0.750 & 1.000 & 0.997 \\
\hline & - & 0 & 605 & & & & & \\
\hline \multirow[t]{2}{*}{ Norovirus } & + & 31 & 7 & 1.000 & 0.988 & 0.816 & 1.000 & 0.982 \\
\hline & - & 0 & 575 & & & & & \\
\hline \multirow[t]{2}{*}{ Rotavirus } & + & 55 & 2 & 1.000 & 0.996 & 0.964 & 1.000 & 0.990 \\
\hline & - & 0 & 556 & & & & & \\
\hline \multirow[t]{2}{*}{ Adenovirus } & + & 9 & 3 & 1.000 & 0.995 & 0.750 & 1.000 & 0.993 \\
\hline & - & 0 & 601 & & & & & \\
\hline \multirow[t]{2}{*}{ Astrovirus } & + & 4 & 0 & 1.000 & 1.000 & 1.000 & 1.000 & 0.997 \\
\hline & - & 0 & 609 & & & & & \\
\hline
\end{tabular}

The outcomes of DP-HMGS and sequencing-based DP detection methods were compared. Sensitivity $=$ TP $/(T P+F N) \times 100$, Specificity $=$ TN $/(T N+F P) \times 100 ;$ PPV and NPV were calculated as follows: PPV $=T P / P, N P V=T N / N$. Notably, the sensitivity of DP-HMGS is high and comparable to that of sequencing for virtually all DP species

$D P-H M G S$ diarrheal pathogens high-throughput multiple genetic detection system, $P P V$ positive predict value, NPV negative predict value

and EIEC, albeit relatively rare, exhibited a very strong predilection for occurring in female patients (Table 3).

The superior potential of DP-HMGS to detect polymicrobials reveals an unexpectedly high incidence of multifactorial diarrhoea

The subsequent evaluation of 613 faecal specimens for polymicrobial infections revealed that approximately onethird of the confirmed infectious diarrhoeas were polymicrobial, which was unexpected (Fig. 7a). Interestingly, some DPs tended to exhibit polymicrobial infections more often than they exhibited individual infections, such as EPEC, EAEC, EIEC, E. coli O157, EHEC, S. typhimurium, Vibrio, Shigella and $Y$. enterocolitica. As shown in Additional file 6: Table S3, the most commonly detected polymicrobial infections were EPEC and Vibrio, EPEC and EAEC, EPEC and S. typhimurium, and EPEC and $C$. jejuni. In addition, there were some triple bacterial infections, such as EPEC, ETEC and EAEC and EPEC, EAEC and S. typhimurium. In addition to the multi-bacterial 


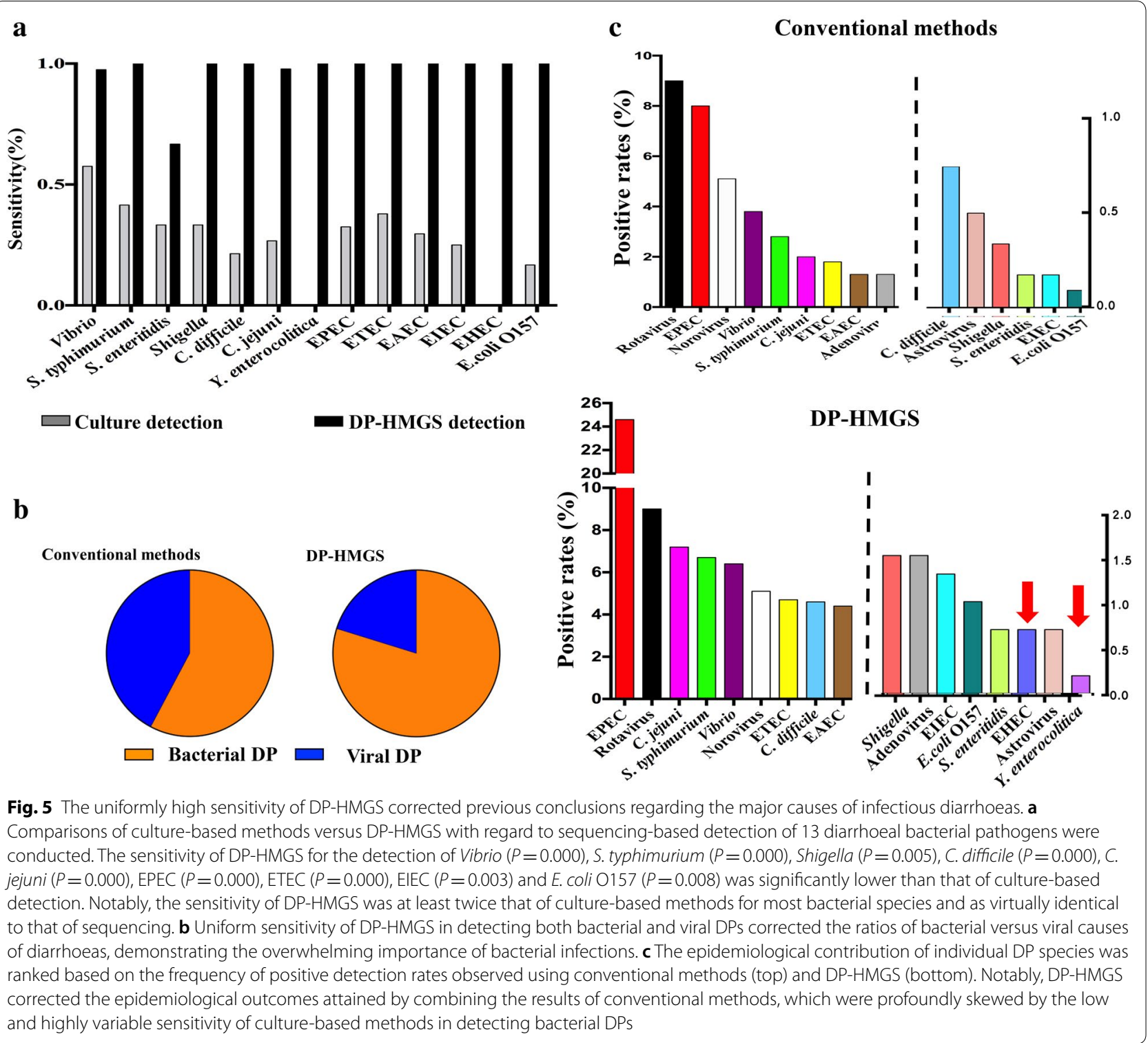

infections, we also observed polymicrobial infections with bacteria and viruses, e.g., C. difficile and rotavirus; EPEC and rotavirus; and EPEC, norovirus and Vibrio. We found that among most of the bacterial DPs, the number of polymicrobial infections was as high as or greater than the number of single infections. In particular, for pathogenic $E$. coli, the number of polymicrobial infections was much greater than that of single infections. In contrast, the viral infections exhibited a high prevalence of single DPs and a low number of co-detected DPs, especially in rotavirus- and norovirus-infected patients (Fig. 7b). Finally, we observed that the frequency of polymicrobial infections was greatest among the 20-39-year-old age group (Fig. 7c), members of which were significantly more likely to develop polymicrobial infections than the members of the 0-19-year-old $(P=0.015)$ and 40-59-year-old patient groups $(P=0.016)$. Thus, we revealed unexpectedly high rates of polymicrobial infections in the studied patient population, particularly in patients in the $20-39$-year-old age group.

\section{Discussion}

Diarrhoeal infections represent a class of highly infectious diseases that rapidly spread and significantly impact the health of large populations, with the most severe cases leading to death [5]. Causes of gastroenteritis are predominantly infectious, triggered by multiple classes of bacterial, viral and parasitic pathogens or other noninfectious factors [27]. Detection of DPs in these cases is 


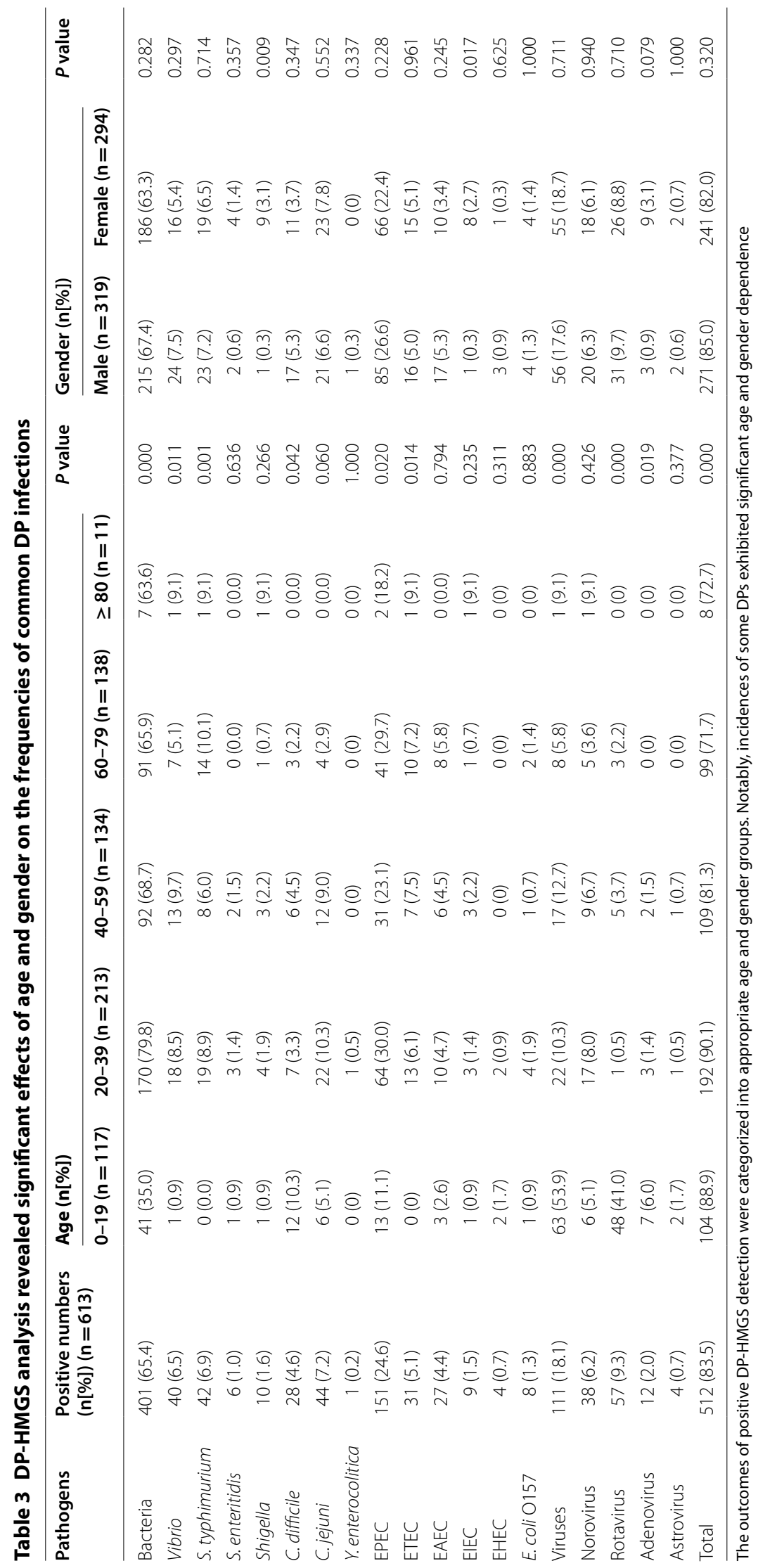




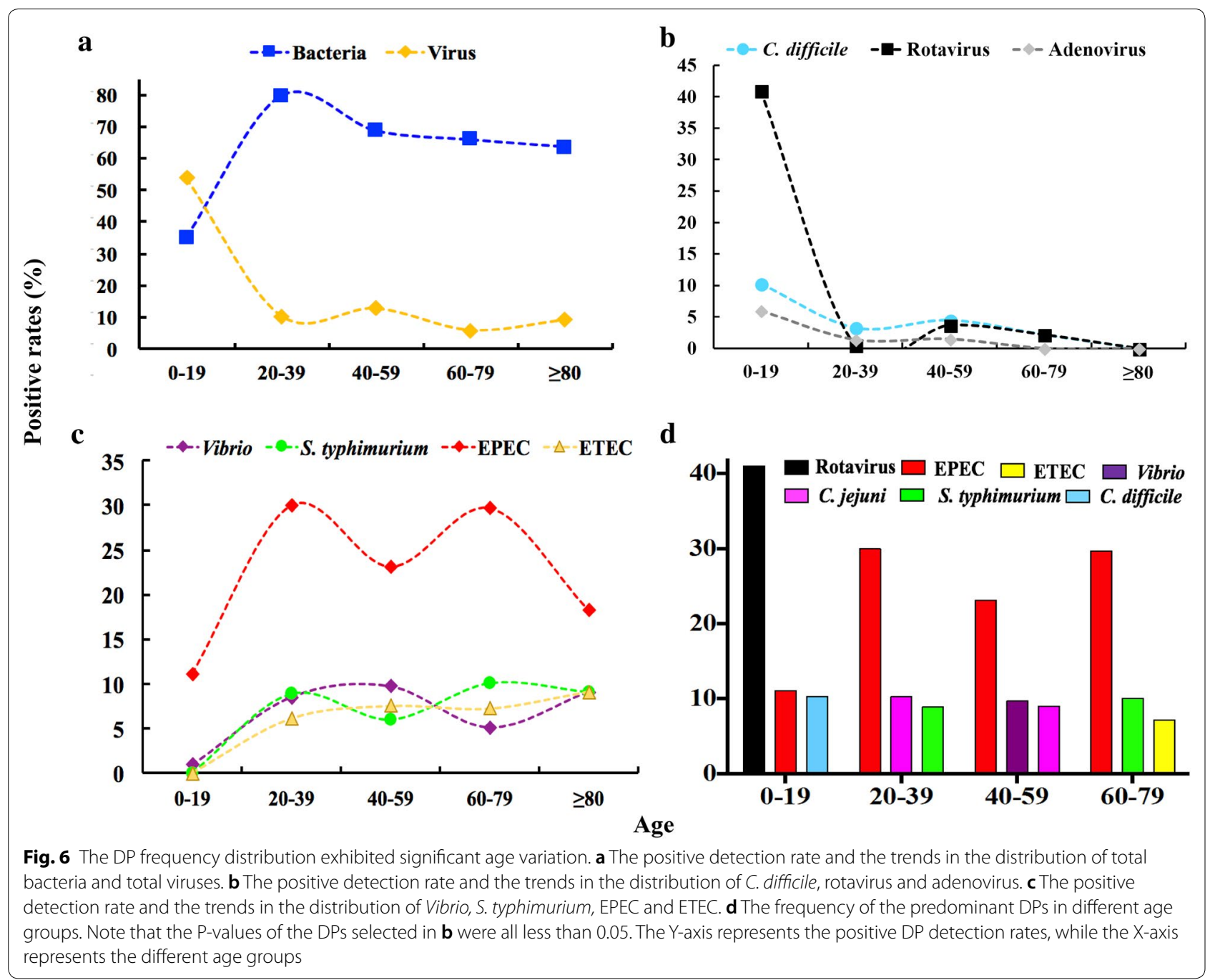

not routinely conducted or is conducted using conventional detection methods, such as culture-based methods and singleplex real-time PCR. These methods are relatively time consuming, costly (due to the need for the application of multiple approaches), and labour intensive and are usually limited to the detection of a single pathogen or a group of closely related pathogens per test $[28,29]$. In this study, we established and optimized a rapid, sensitive, specific and well-controlled DP identification and screening assay-DP-HMGS - which allowed the detection of 19 classes of pathogenic DPs simultaneously in faecal specimens. Systematic analysis of 613 clinical specimens with this highly sensitive and specific DP-HMGS assay revealed that (1) the DP-HMGS method was more sensitive and could detect more pathogenic bacteria than the culture-based method while maintaining sensitivity levels comparable to those of single PCRbased detection; (2) several major aetiological agents remained frequently underdiagnosed as important causes of acute infectious diarrhoeas when assessed solely using conventional methods, leading to incorrect conclusions regarding major causes of infectious diarrhoeas; (3) the DP frequency distribution detected by DP-HMGS exhibited significant age variation; and (4) approximately $1 / 3$ of the cases of infectious diarrhoeas were co-induced by multiple pathogens, with some DPs preferentially occurring as co-infecting agents.

Culture-based methods are the most conventional methods and continue to be commonly used for the detection of bacterial causes of diarrhoea as a "gold standard" for aetiological diagnosis in a vast majority of health centres. However, culture-based methods have several significant limitations that restrict the use of these methods as the "first line" of DP screening [28, 29]. These limitations include the requirement of up to several days of growth before analysis, requirement of variable media and culture conditions for various species, problems associated with overgrowth of non-pathogenic bacteria that are 


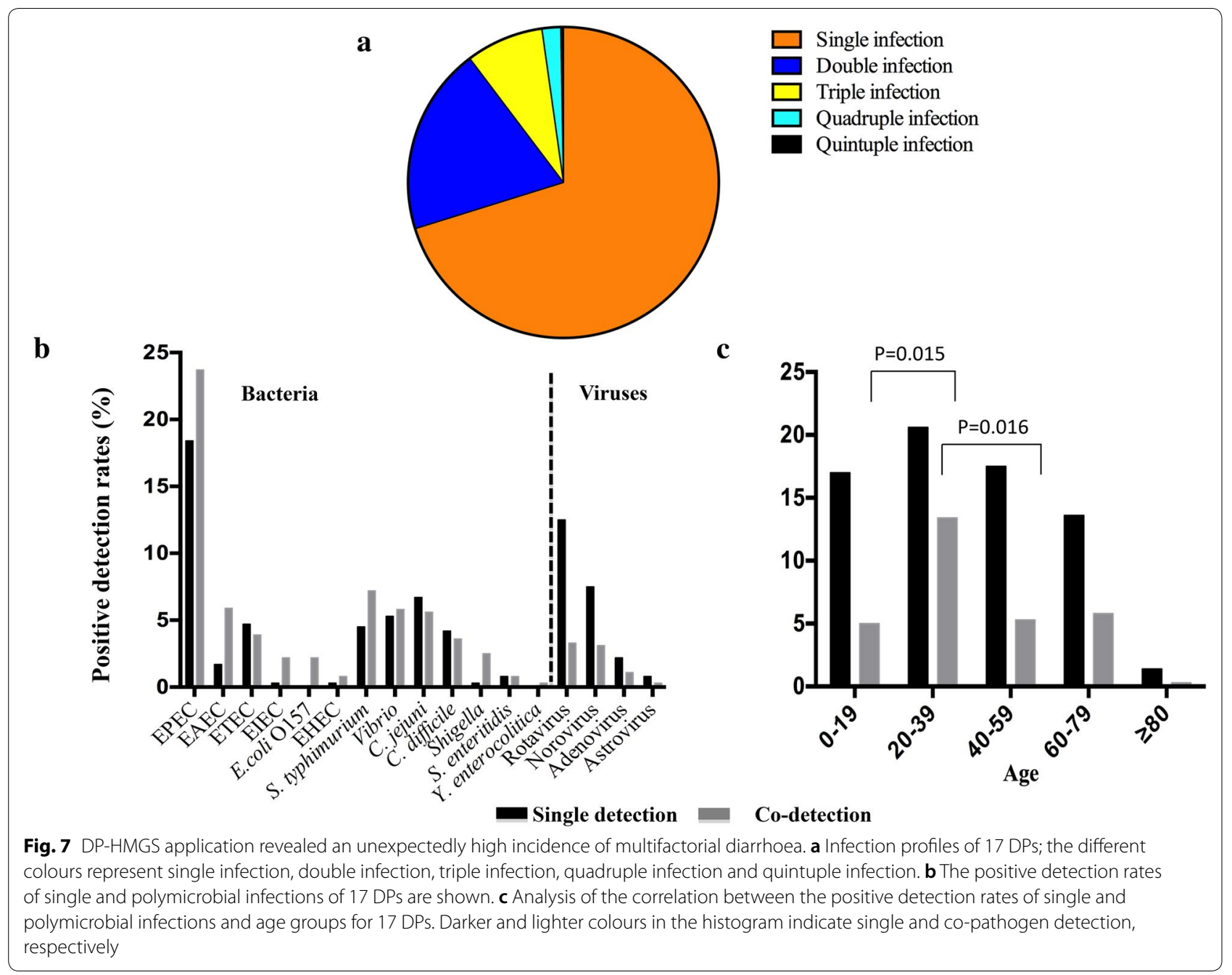

abundant in the gut, and limited sensitivity due to technical limitations of incubation environments. Finally, a number of strictly anaerobic but important bacterial species, such as $C$. difficile are difficult to be cultured under routine laboratory conditions due to the requirement of specific equipment [30,31]. Detection of viruses is commonly conducted by singleplex real-time PCR. However, the use of individual PCRs to screen multiple pathogens is tedious and costly [29, 32,33]. Our present study demonstrates that the detection levels for most bacterial pathogens using culture-based methods alone were unacceptably low. Indeed, for most pathogens, the detection levels were $30 \%$ or lower (Table 1). Furthermore, low and non-uniform culture detection rates resulted in a significant bias, with the epidemiological data showing a very high impact of viral infection and greatly underestimating that of bacteria (Fig. 5). DP-HMGS allowed us to overcome the significant disparity in the sensitivities of the conventional detection methods, bringing the accuracy of the epidemiological map of DPs to the level obtained by sequencing methods (Table 2, Fig. 5). The major advantages of the DP-HMGS platform that allowed its successful implementation were high sensitivity, specificity and accuracy for the identification of DP and, most importantly, a relatively uniform performance for all DPs. One potential drawback of DNA/RNA-based molecular detection methods (including DP-HMGS) is that these methods do not reveal whether an infectious agent is viable. However, considering the detection limit $\left(10^{2}-10^{3}\right.$ copies/ $\mu \mathrm{L}$ ), DP-HMGS detected only significantly abundant microbes in the GI tract, in turn strongly suggesting the association of the microbes with the disease. Further studies are needed to definitively address whether the detection of microbes at this level always signifies the presence of viable microbes in the GI tract.

In terms of epidemiological findings, we showed that EPEC was the DP most frequently associated with infectious diarrhoeas in Shanghai, accounting for $24.6 \%$ 
of cases (Table 3); this finding was consistent with previous reports from China [34] and Singapore [35]. In contrast, a report by Moreno et al. concluded that EAEC rather than EPEC was the major cause of diarrhoea [36]. While its relative contributions to diarrhoea epidemiology have often been inconsistent between reports [37, 38], EPEC continues to be the most prevalent type of pathogenic $E$. coli found in industrialized countries $[39,40]$. A strong association between EPEC and diarrhoea in children has been reported [39], and yet, we also found a relatively high prevalence of EPEC in older patients in our study (Fig. 6c). In the study of the age distribution of pathogens, the predominant DP frequencies varied among all age groups, as exemplified by the "top 3" DPs in each age interval (Fig. 6d), which might be attributed to the different lifestyles, food preferences and immune statuses of the patients in different age groups. However, these disparities were likely influenced by different research periods and the variety of methods applied for detection. Rotavirus was most frequently found and highly prevalent among young patients (0-19 ages) but less prevalent in other age groups, which was consistent with the results of most epidemiological studies $[17,41]$. Another infection that predominantly impacted patients $\leq 19$ years old was pathogenic $C$. difficile. This result is consistent with a report by Buss et al. that showed a high proportion of C. difficile in the infectious DPs detected from paediatric faecal specimens using the FilmArray gastrointestinal panel [42]. The clinical practice guidelines for $C$. difficile infection in adults and children do not recommend testing for $C$. difficile in children less than 2 years old unless other causes of disease have been explicitly excluded [43]. In our study, we specifically detected pathogenic $C$. difficile in 0-2-year-old patients with diarrhoea symptoms without a confirmed clinical diagnosis of other causes of disease. These outcomes provided useful diagnostic clues, suggesting that in most of these cases, the major cause of diarrhoea was pathogenic $C$. difficile. Thus, future studies are needed to establish whether the detection of pathogenic $C$. difficile in young children in these circumstances is important for clinical diagnosis and treatment.

The final important finding in our study was a high frequency of polymicrobial infection with two or more DPs, relative to previously reported data $[44,45]$, which demonstrated another level of complexity in determining the aetiology of diarrhoea, which could be offset by the use of multiplex detection systems such as DP-HMGS. Because of the limitations of conventional methods, polymicrobial infections usually go undetected, and none of the polymicrobial infections could be identified by culture-based methods in our study [46]. The polymicrobial infections identified by DP-HMGS accounted for $1 / 3$ of the positive specimens detected (Fig. 7a), showing that the E. coli subgroups were much more common in polymicrobial infections than as the cause of single DP infections (Fig. 7b). This result may be due to the E. coli pathogroup requiring partner pathogens to cause severe diarrhoeal disease [46-48]. Among the polymicrobial infections, we found that EPEC and Vibrio presented the highest ratio (Additional file 6: Table S3). One possible reason for this observation could be that the isolation rate of EPEC was the highest (24.6\%), and Vibrio was the most frequently isolated pathogen from seawater and seafood, and the consumption of contaminated seafood appears to be one of the major causes of acute diarrhoea in Shanghai [49]. Finally, we noticed that the proportion of polymicrobial infections in the 20-39-yearold age group was significantly higher than that in the 40-59-year-old age group (Fig. 7c), which may be attributed to the different lifestyles of younger adults, including more frequent social activity, travelling and moving than the older group.

However, these findings regarding polymicrobial infections could be a result of colonization by another DP because differentiation between colonization and true polymicrobial infections is relatively difficult (regardless of the detection methods) [50]. One advantage of DP-HMGS was that specific pathogenic gene sequences were used for the detection of C. difficile, S. typhimurium, Shigella and E. coli pathogenic strains (EPEC, ETEC, EAEC, EIEC, EHEC and E. coli O157). Thus, DP-HMGS could be used to distinguish between colonizing and pathogenic bacteria. Nevertheless, for the remaining DPs, which did not have specific pathogenicity-associated genes, colonization could not be distinguished from an active pathogenic process.

\section{Conclusions}

In summary, we developed and optimized a rapid, sensitive, highly specific multiplex DP identification system, namely, DP-HMGS, which allowed the detection of 19 classes of viral and bacterial DPs via a single test. Systematic analysis of 613 clinical specimens with the DP-HMGS assay significantly remapped the aetiology of infectious diarrhoeas in Shanghai. This result included the detection of underdiagnosed or previously undetected bacterial pathogens and unexpectedly high levels of infections co-induced by multiple DPs. Our study documents that DP-HMGS provides a highly effective and time-saving alternative for the clinical diagnosis of faecal specimens. We propose that the widespread use of such assays on a global scale would be helpful to update and correct the epidemiological map of major causes of diarrhoeal infections and promote rational treatments of infectious diarrhoeas. 


\section{Additional files}

Additional file 1: Table S1. Species-specific genes, primer sequences and product sizes for DPs in DP-HMGS. The designed primer sets, speciesspecific genes and the corresponding amplicon sizes for the molecular detection of 6 classes of viral and 13 classes of bacterial DPs as well as 3 quality controls for the DP-HMGS assay.

Additional file 2: Table S2. Conventional PCR primer sequences and product sizes for Sanger sequencing. The designed primer sets and the corresponding amplicon sizes for molecular detection of 6 classes of viral and 13 classes of bacterial DPs as well as 3 quality controls for Sanger sequencing.

Additional file 3: Figure S1. Primers designed for DP-HMGS effectively detect DPs in individual PCRs. In the DP-HMGS assay reaction system with all DP signature primers present, the nucleic acid templates for each of the pathogens were tested individually. Each class of diarrhoeal pathogens was successfully detected by DP-HMGS. The specific peaks individually appeared at 209 bp for C. jejuni (A), 136 bp for Shigella (B), 202 bp for C. difficile (C), 159 bp for HASV (D), 226 bp for norovirus (E), 190 bp for ETEC (F), 152 bp for EHEC (G), 251 bp for EPEC (H), 292 bp for EAEC (I), 129 bp for EIEC (J), 308 bp for rotavirus (K), 120 bp for $S$. enteritidis (L), 113 bp for $S$. typhimurium (M), 196 bp for Vibrio (N), 145 bp for HADV (O), 165 bp for $Y$. enterocolitica (P) and 218 bp for E. coli O157 (Q). Notably, the peaks in A-Q at $313 \mathrm{bp}$ were for the IC, and all gene targets were specifically amplified without non-specific amplification by individual PCR assays.

Additional file 4: Figure S2. The DP-HMGS assay was highly specific for DP identification. (A) In the positive control, DP-HMGS detection of DP was conducted by simultaneous detection of the plasmid templates shown here for 17 pathogens and 3 quality controls. All the targets (from left to right: hum_RNA, S. typhimurium, S. enteritidis, EIEC, Shigella, HADV, EHEC, HASV, Y. enterocolitica, ETEC, Vibrio, C. difficile, C. jejuni, E. coli O157, norovirus, hum_DNA, EPEC, EAEC, rotavirus, IC) could be specifically detected by DP-HMGS. (B-H) DP-HMGS assay results obtained using 7 individual plasmid templates from H. pylori, E. coli DH5a, P. aeruginosa, S. aureus, P. shigelloides, sapovirus, and non-pathogenic $E$. coli showed no pathogenspecific peak. (I) DP-HMGS assay results obtained by using $\mathrm{ddd}_{2} \mathrm{O}$ as a DNA template showed no pathogen-specific peak. DP-specific genes produced signals only in the positive controls, not in the negative control, which consisted of pathogens that could potentially interfere with specific pathogen detection. Notably, the peaks in B-H at 313 bp were for the IC.

Additional file 5: Figure S3. DP-HMGS could directly detect specific microbial signatures in faecal specimens. (A) The human faecal specimen with a specific DP infection produced a C. difficile-specific peak at 202 bp, as well as specific peaks for the human internal RNA control, human internal DNA control and internal positive test control at $106 \mathrm{bp}, 233 \mathrm{bp}$ and $313 \mathrm{bp}$, respectively. (B) The faecal specimens from non-infectious diarrhoea patients produced only the specific peaks of Hum_RNA, Hum_DNA and IC at 106 bp, 233 bp and 313 bp, respectively. (C) dd ${ }_{2} \mathrm{O}$ showed only the specific peak for the IC at $313 \mathrm{bp}$

Additional file 6: Table S3. Frequency of the combinations of polymicrobial infections. The combinations and the percentages of the most common polymicrobial DP infections include double infections, triple infections, multi-bacterial infections and bacterial/viral polymicrobial infections.

\section{Abbreviations}

DP: diarrhoeal pathogens; DP-HMGS: high-throughput multiplex genetic detection system for diarrhoeal pathogen detection; HASV: human astrovirus; NorV: norovirus II; HADV: human adenovirus; RoVA: rotavirus A; RoVB: rotavirus B; RoVC: rotavirus C; C. jejuni: Campylobacter jejuni; C. difficile: Clostridium difficile; S. enteritidis: Salmonella enteritidis; S. typhimurium: Salmonella typhimurium; Y. enterocolitica: Yersinia enterocolitica; H. pylori: Helicobacter pylori; S. aureus: Staphylococcus aureus; ETEC: enterotoxigenic E. coli; EHEC: enterohemorrhagic E. coli; EPEC: enteropathogenic E. coli; EAEC: enteroaggregative E. coli; EIEC: enteroinvasive E. coli.

\section{Authors' contributions}

SW, FY, DL and JQ contributed equally to this work. ZB, MAO, HZ and YZ contributed to the design and coordinated the study. SW, YW, YZ, YM, LX and $J C$ designed the primers and optimized the conditions of the DP-HMGS assay. $\mathrm{DL}, \mathrm{WH}, \mathrm{LJ}, \mathrm{YF}$ and $\mathrm{FZ}$ collected and verified the bacteria and viruses. SW, $\mathrm{DL}$, $\mathrm{MK}, \mathrm{ZB}, \mathrm{MAO}, \mathrm{HZ}$ and $\mathrm{YZ}$ wrote the manuscript. All authors read and approved the final manuscript.

\section{Author details}

${ }^{1}$ Department of Laboratory Medicine, Huadong Hospital, Affiliated with Fudan University, Shanghai 200040, China. ${ }^{2}$ Shanghai Key Laboratory of Clinical Geriatric Medicine, Shanghai 200040, China. ${ }^{3}$ Research Center on Aging and Medicine, Fudan University, Shanghai 200040, China. ${ }^{4}$ Department of Clinical Laboratory, Shanghai Tongji Hospital, Tongji University School of Medicine, Shanghai 200065, China. ${ }^{5}$ Department of Laboratory Medicine, Renji Hospital, School of Medicine, Shanghai Jiaotong University, Shanghai 200135, China. ${ }^{6}$ Ningbo HEALTH Gene Technologies Co., Ltd., Ningbo, China. ${ }^{7}$ Shanghai ABSciex Analytical Instrument Trading Co., Ltd., Shanghai, China. ${ }^{8}$ Department of Gastroenterology, Gerontology Institute of Shanghai, Affiliated with Huadong Hospital, Affiliated with Fudan University, Shanghai 200040, China. ${ }^{9}$ Division of Pulmonary and Critical Care Medicine, Department of Internal Medicine, University of Michigan Health System and Research Service, VA Ann Arbor Health Systems, Ann Arbor, MI, USA.

\section{Acknowledgements}

The authors are grateful to David M. Aronoff (Vanderbilt University Medical Center), Changqing Yang (Department of Gastroenterology, Tongji Hospital, affiliated with Shanghai Tongji University), Min Chen (Shanghai Municipal Center for Disease Control \& Prevention), and Min Li (Department of Laboratory Medicine, Renji Hospital, School of Medicine, Shanghai Jiaotong University) for their valuable guidance and support in this study.

\section{Competing interests}

The authors declare that they have no competing interests.

\section{Availability of data and materials}

All data generated or analysed during this study are included in this published article and its additional files.

\section{Consent for publication}

Not applicable.

\section{Ethics approval and consent to participate}

This study was carried out in accordance with the recommendations of the Ethics Committee for Human Studies of Huadong Hospital and registered under Ethics Approval Number 2013-077 with written informed consent from all subjects. All subjects provided written informed consent following the principles outlined in the Declaration of Helsinki for all human experimental investigations. The protocol was approved by the Ethics Committee for Human Studies of Huadong Hospital.

\section{Funding}

This work was supported by the Shanghai Shenkang Hospital Development Center "New frontier technology joint research project" (Grant No.: SHDC12015107), National Natural Science Foundation Grant of China (Grant No.: 81602072), Shanghai Science and Technology Committee "Lead project" (Grant No.: 16411968000), Ministry of Science and Technology "The National High Technology Research and Development Program of China (863 Program)" (Grant No.: 2015AA021 107-019), Shanghai Sailing Program (Grant No.: 2017YF1405000), and Shanghai Science and Technology Committee "Scientific and technological innovation action projects" (Grant No.: 18411950800). Olszewski MA was supported by the VA Merit Grant (Grant No.: 101BX000656) and VA Research Career Scientist Award (Grant No:: 1IK6BX003615-01). Funding was also received from the Shanghai Municipal Commission of Health and Family Planning, key developing disciplines (Grant No.: 2015ZB0501), and the Songjiang Shanghai public health system construction plan of action for 3 years-community crowd acute respiratory infection monitoring system construction (Grant No.: 605414) 


\section{Publisher's Note}

Springer Nature remains neutral with regard to jurisdictional claims in published maps and institutional affiliations.

Received: 17 May 2018 Accepted: 27 August 2018

Published online: 11 September 2018

\section{References}

1. Black RE, Cousens S, Johnson HL, Lawn JE, Rudan I, Bassani DG, et al. Global, regional, and national causes of child mortality in 2008: a systematic analysis. Lancet. 2010;375(9730):1969-87.

2. Farthing $M$, Salam MA, Lindberg G, Dite P, Khalif I, Salazar-Lindo E, et al. Acute diarrhea in adults and children: a global perspective. J Clin Gastroenterol. 2013;47(1):12-20

3. Hou FQ, Wang Y. LI J, Wang GQ, Liu Y. Management of acute diarrhea in adults in China: a cross-sectional survey. BMC Public Health. 2013;13:41.

4. Lozano R, Naghavi M, Foreman K, Lim S, Shibuya K, Aboyans V, et al. Global and regional mortality from 235 causes of death for 20 age groups in 1990 and 2010: a systematic analysis for the Global Burden of Disease Study 2010. Lancet. 2012;380(9859):2095-128.

5. Patten SB. The global burden of disease 2010 update: keeping mental health in the spotlight. Epidemiol Psychiatr Sci. 2014;23(3):255-7.

6. Havelaar AH, Kirk MD, Torgerson PR, Gibb HJ, Hald T, Lake RJ, et al. World Health Organization global estimates and regional comparisons of the burden of foodborne disease in 2010. PLoS Med. 2015;12(12):e1001923.

7. Freeman K, Tsertsvadze A, Taylor-Phillips S, McCarthy N, Mistry H, Manuel $\mathrm{R}$, Mason J. Agreement between gastrointestinal panel testing and standard microbiology methods for detecting pathogens in suspected infectious gastroenteritis: test evaluation and meta-analysis in the absence of a reference standard. PLoS ONE. 2017;12(3):e173196.

8. Platts-Mills JA, Operario DJ, Houpt ER. Molecular diagnosis of diarrhea: current status and future potential. Curr Infect Dis Rep. 2012:14(1):41-6.

9. Manual clinical microbiology, 11th Ed. Washington: American Society for Microbiology (ASM) Press; 2015.

10. Clinical microbiology examination. Beijing: People's Medical Publishing House; 2015.

11. Binnicker MJ. Multiplex Molecular Panels for Diagnosis of Gastrointestinal Infection. Performance, Result Interpretation, and Cost-Effectiveness. J Clin Microbiol. 2015:53(12):3723-8.

12. Qu M, Lv B, Zhang X, et al. Prevalence and antibiotic resistance of bacterial pathogens isolated from childhood diarrhea in Beijing, China (2010-2014). Gut Pathog. 2016;8:31.

13. Guan $\mathrm{H}$, Zhang J, Xiao Y, et al. Evaluation of PCR based assays for the improvement of proportion estimation of bacterial and viral pathogens in diarrheal surveillance. Front Microbiol. 2016;7:386.

14. Tian L, Zhu X, Chen Z, Liu W, Li S, Yu W, et al. Characteristics of bacterial pathogens associated with acute diarrhea in children under 5 years of age: a hospital-based cross-sectional study. BMC Infect Dis. 2016;16:253.

15. Zhu XH, Tian L, Cheng ZJ, Liu WY, Li S, Yu WT, et al. Viral and bacterial etiology of acute diarrhea among children under 5 years of age in Wuhan China. Chin Med J (Engl). 2016;129(16):1939-44.

16. Jia L, Lin C, Gao Z, Qu M, Yang J, Sun J, et al. Prevalence and factors associated with different pathogens of acute diarrhea in adults in Beijing, China. J Infect Dev Ctries. 2016;10(11):1200-7.

17. Lamberti LM, Walker CLF, Black RE. Systematic review of diarrhea duration and severity in children and adults in low- and middle-income countries. BMC Public Health. 2012;12(1):276

18. Thielman NM, Guerrant RL. Clinical practice. Acute infectious diarrhea. New Engl J Med. 2004;350(1):38-47.

19. Zhang Y, Zhao F, Kong M, Wang S, Nan L, Hu B, et al. Validation of a highthroughput multiplex genetic detection system for Helicobacter pylori identification, quantification, virulence, and resistance analysis. Front Microbiol. 2016;7:1401.

20. Zhang Y, Zhao Y, Ding K, Wang X, Chen X, Liu Y, et al. Analysis of bacterial pathogens causing acute diarrhea on the basis of sentinel surveillance in Shanghai, China, 2006-2011. Jpn J Infect Dis. 2014;67(4):264-8.

21. Varela G, Batthyany L, Bianco MN, Perez W, Pardo L, Algorta G, et al. Enteropathogens associated with acute diarrhea in children from households with high socioeconomic level in uruguay. Int J Microbiol. 2015;2015:592953.

22. Xiao-Ling YU, Zhang BJ, Chun-Xiang LU. Analysis of the surveillance results of infectious diarrhea pathogens in Chongming County in 2014. Chin J Health Lab Technol. 2016:26(18):2682-5.

23. Riddle MS, DuPont HL, Connor BA. ACG clinical guideline: diagnosis, treatment, and prevention of acute diarrheal infections in adults. Am J Gastroenterol. 2016;111(5):602-22.

24. Krokan HE, Otterlei M, Nilsen H, Kavli B, Skorpen F, Andersen S, et al. Properties and functions of human uracil-DNA glycosylase from the UNG gene. Prog Nucleic Acid Res Mol Biol. 2001;68:365-86.

25. Mu W, Lu HM, Chen J, Li S, Elliott AM. Sanger confirmation is required to achieve optimal sensitivity and specificity in next-generation sequencing panel testing. J Mol Diagn. 2016;18(6):923-32.

26. Baudhuin LM, Lagerstedt SA, Klee EW, Fadra N, Oglesbee D, Ferber MJ. Confirming variants in next-generation sequencing panel testing by Sanger sequencing. J Mol Diagn. 2015;17(4):456-61.

27. Hilmarsdottir I, Baldvinsdottir GE, Harethardottir $\mathrm{H}$, Briem $\mathrm{H}$, Sigurethsson SI. Enteropathogens in acute diarrhea: a general practice-based study in a Nordic country. Eur J Clin Microbiol Infect Dis. 2012;31(7):1501-9.

28. Lee JY, Cho SY, Hwang H, Ryu JY, Lee J, Song ID, et al. Diagnostic yield of stool culture and predictive factors for positive culture in patients with diarrheal illness. Medicine (Baltimore). 2017;96(30):e7641.

29. O'Connor O, Cooke RP, Cunliffe NA, Pizer B. Clinical value of stool culture in paediatric oncology patients: hospital evaluation and UK survey of practice. J Hosp Infect. 2017;95(1):123-5.

30. Shin BM, Lee EJ. Comparison of ChromID agar and Clostridium difficile selective agar for effective isolation of $C$. difficile from stool specimens. Ann Lab Med. 2014:34(1):15-9.

31. Rodriguez C, Warszawski N, Korsak N, Taminiau B, Van Broeck J, Delmee $\mathrm{M}$, et al. Laboratory identification of anaerobic bacteria isolated on Clostridium difficile selective medium. Acta Microbiol Immunol Hung. 2016;63(2):171-84

32. Amar CF, East CL, Gray J, Iturriza-Gomara M, Maclure EA, McLauchlin J. Detection by PCR of eight groups of enteric pathogens in 4627 faecal samples: re-examination of the English case-control Infectious Intestinal Disease Study (1993-1996). Eur J Clin Microbiol Infect Dis. 2007:26(5):311-23.

33. Lehours P, Siffre E, Megraud F. DPO multiplex PCR as an alternative to culture and susceptibility testing to detect Helicobacter pylori and its resistance to clarithromycin. BMC Gastroenterol. 2011;11:112.

34. Zhang Z, Lai S, Yu J, et al. Etiology of acute diarrhea in the elderly in China: a six-year observational study. PLoS ONE. 2017;12(3):e0173881.

35. Ahmad K, Mitra M, Mehdi R, Ahmad P. Characterization of virulence genes in typical and atypical enteropathogenic Escherichia coli. Sci Res Essays. 2011;31:6600-5.

36. Moreno AC, Filho AF, Gomes Tdo A, Ramos ST, Montemor LP, Tavares VC, et al. Etiology of childhood diarrhea in the northeast of Brazil: significant emergent diarrheal pathogens. Diagn Microbiol Infect Disf. 2010;66(1):50-7.

37. Mitra M, Ahmad P, Mehdi R, Hosein A, Ahmad K. Multiple drug resistance of enteropathogenic Escherichia coli isolated from children with diarrhea in Kashan. Iran. African Journal of Microbiology Research. 2011;5(20):3305-9.

38. Prere MF, Bacrie SC, Baron O, Fayet O. Bacterial aetiology of diarrhoea in young children: high prevalence of enteropathogenic Escherichia coli (EPEC) not belonging to the classical EPEC serogroups. Pathol Biol (Paris). 2006:54(10):600-2.

39. Ochoa TJ, Barletta F, Contreras C, Mercado E. New insights into the epidemiology of enteropathogenic Escherichia coli infection. Trans R Soc Trop Med Hyg. 2008;102(9):852-6.

40. Santona S, Diaz N, Fiori PL, Francisco M, Sidat M, Cappuccinelli P, et al. Genotypic and phenotypic features of enteropathogenic Escherichia coli isolated in industrialized and developing countries. J Infect Dev Ctries. 2013;7(3):214-9.

41. Liu L, Yuan Q, You Z, Zhao L, Jia L, Dong H. Epidemiological aspects of rotavirus and adenovirus in hospitalized children with diarrhea: a 5-year survey in Beijing. BMC Infect Dis. 2016;16(1):508.

42. Buss SN, Leber A, Chapin K, Fey PD, Bankowski MJ, Jones MK, et al. Multicenter evaluation of the BioFire FilmArray gastrointestinal panel 
for etiologic diagnosis of infectious gastroenteritis. J Clin Microbiol. 2015;53(3):915-25.

43. McDonald LC, Gerding DN, Johnson S, Bakken JS, Carroll KC, Coffin SE, et al. Clinical Practice Guidelines for Clostridium difficile Infection in Adults and Children: 2017 Update by the Infectious Diseases Society of America (IDSA) and Society for Healthcare Epidemiology of America (SHEA). Clin Infect Dis. 2018;66(7):987-94.

44. Mason J, Iturriza-Gomara M, O'Brien SJ, Ngwira BM, Dove W, Maiden MC, et al. Campylobacter infection in children in Malawi is common and is frequently associated with enteric virus co-infections. PLoS ONE. 2013;8(3):e59663.

45. Zhang SX, Zhou YM, Xu W, Tian LG, Chen JX, Chen SH, et al. Impact of co-infections with enteric pathogens on children suffering from acute diarrhea in southwest China. Infect Dis Poverty. 2016;5(1):64.

46. Julian TR. Environmental transmission of diarrheal pathogens in low and middle income countries. Environ Sci Process Impacts. 2016;18(8):944-55.
47. Mehla K, Ramana J. DBDiaSNP: an open-source knowledgebase of genetic polymorphisms and resistance genes related to diarrheal pathogens. OMICS. 2015;19(6):354-60.

48. Huang SH, Lin YF, Tsai MH, Yang S, Liao ML, Chao SW, et al. Detection of common diarrhea-causing pathogens in northern Taiwan by multiplex polymerase chain reaction. Medicine (Baltimore). 2018;97(23):e11006.

49. Zhao R, Yan S, Liu M, Wang B, Hu D, Guo D, et al. Seafood consumption among Chines coastal residents and health risk assessment of heavy metals in seafood. Environ Sci Pollut Res Int. 2016;23(16):16834-44.

50. Fauchier T, Hasseine L, Gari-Toussaint M, Casanova V, Marty PM, Pomares C. Detection of Pneumocystis jirovecii by quantitative PCR to differentiate colonization and pneumonia in immunocompromised HIV-positive and HIV-negative patients. J Clin Microbiol. 2016;54(6):1487-95.
Ready to submit your research? Choose BMC and benefit from:

- fast, convenient online submission

- thorough peer review by experienced researchers in your field

- rapid publication on acceptance

- support for research data, including large and complex data types

- gold Open Access which fosters wider collaboration and increased citations

- maximum visibility for your research: over $100 \mathrm{M}$ website views per year

At BMC, research is always in progress.

Learn more biomedcentral.com/submissions 